\title{
A Novel Approach for the Efficient Computation of 1-D and 2-D Summations
}

\author{
E. Pınar Karabulut, Member, IEEE, Vakur B. Ertürk, Member, IEEE, Lale Alatan, Member, IEEE, \\ S. Karan, Burak Alisan, and M. I. Aksun, Senior Member, IEEE
}

\begin{abstract}
A novel computational method is proposed to evaluate 1-D and 2-D summations and integrals which are relatively difficult to compute numerically. The method is based on applying a subspace algorithm to the samples of partial sums and approximating them in terms of complex exponentials. For a convergent summation, the residue of the exponential term with zero complex pole of this approximation corresponds to the result of the summation. Since the procedure requires the evaluation of relatively small number of terms, the computation time for the evaluation of the summation is reduced significantly. In addition, by using the proposed method, very accurate and convergent results are obtained for the summations which are not even absolutely convergent. The efficiency and accuracy of the method are verified by evaluating some challenging 1-D and 2-D summations and integrals.
\end{abstract}

Index Terms-Acceleration techniques, cylindrically stratified media, Green's functions, numerical methods, periodic structures, planar layered structures, Sommerfeld integrals.

\section{INTRODUCTION}

I N MANY science and engineering applications, relatively difficult and usually infinite summations with quite complicated functions need to be computed numerically. These applications may include, in addition to many others, the estimation of electrostatic interactions in molecular dynamics [1], [2], and the calculations of Green's functions, periodic Green's functions in free space, and layered media in electromagnetics (EM) [3]-[5]. The difficulty of the computation usually arises from the highly oscillatory and slowly convergent nature of the summations [6]. For instance in EM, which is the concentration of this study, the analysis of cylindrical geometries may require the computation of infinite summation of cylindrical Hankel- and Bessel-type functions [7], summations of which converge very slowly. Similarly, the function may contain spherical Hankel and Bessel functions together

Manuscript received April 10, 2015; revised December 25, 2015; accepted December 31, 2015. Date of publication January 26, 2016; date of current version March 01, 2016

E. P. Karabulut is with the Department of Electrical and Electronics Engineering, Bahcesehir University, 34349 Istanbul, Turkey (e-mail: eminepinar.karabulut@eng.bahcesehir.edu.tr).

V. B. Ertürk is with the Department of Electrical and Electronics Engineering, Bilkent University, 6800 Ankara, Turkey.

L. Alatan is with the Department of Electrical and Electronics Engineering, Middle East Technical University, 06800 Ankara, Turkey.

S. Karan and B. Alisan are with the Aselsan Electronics Inc., 06370 Ankara, Turkey.

M. I. Aksun is with the Department of Electrical and Electronics Engineering, Koc University, 34450 Istanbul, Turkey.

Color versions of one or more of the figures in this paper are available online at http://ieeexplore.ieee.org.

Digital Object Identifier 10.1109/TAP.2016.2521860 with the Legendre polynomials for spherical geometries [8], [9]. To numerically simulate many structures involving these functions, the computation time for the evaluation of infinite summations/integrals is very crucial as they are usually performed more than once.

In this study, a novel method is proposed to efficiently and accurately compute oscillating and slowly convergent summations and to help assess the nature of convergence or divergence. In principle, the idea of the method is as follows: if one takes a sufficient number of partial sums as a set of data and approximates them as a sum of complex exponentials, the dc term (the residue of the exponential with zero complex pole) of this approximation would be the result of the summation of interest. The method is quite efficient and robust because of the fact that the partial sums of an infinite summation usually exhibit (highly or slowly) oscillatory behavior (due to exponentials related to the phase information of EM propagation), which can be well expressed in terms of complex exponentials. In order to approximate the data as a series of complex exponentials, a subspace approach called the generalized pencil of function (GPOF) method [10] is employed. Moreover, the proposed method can also be used as a convergence/divergence test for infinite summations. This feature stems from the fact that a convergent series of partial sums can be represented by a sum of complex exponentials with a zero exponent (dc term) and exponents with negative real parts only, whereas the divergent ones result in at least one exponent with a positive real part.

It should be noted that the proposed method is also applicable to 1-D and 2-D slowly convergent integrals. Similar to the partial sums in EM problems, since the behavior of the partial integrals of an infinite and convergent integral has an oscillatory and slowly convergent behavior, the dc term of this behavior corresponds to the result of the integral. Therefore, the principle idea is also valid for integrations, and once a sufficient set of partial integrals is obtained, the rest of the algorithm becomes exactly the same.

The method is first applied to slowly convergent 1-D summations, and its results are compared to those obtained by the direct summation (DS) and the Shanks transformation (ST) [11]. Then the accuracy and robustness of the proposed method are demonstrated on a 1-D summation, which is not absolutely convergent. In addition, the method is applied to the Sommerfeld integral tails, which are well-known 1-D integrals with the oscillating and slowly decaying nature, and its performance is compared to the ST and the generalized weighted averages (WA) algorithm [12], [13]. Finally, as a 2-D example, the free-space periodic Green's function (FSPGF) for a doubly 
periodic structure is computed by the proposed method, and its efficiency is compared to the ST and the Ewald summation method [14].

This paper is organized as follows. The theory of the method and the details of its implementation on 1-D and 2-D problems are discussed in Section II. Section III provides the numerical results and conclusion is drawn in Section IV.

\section{Fundamentals of the Method}

In this section, the main idea of the method is demonstrated on 1-D summations, and its extension to 2-D summations is discussed. For the sake of demonstration, let us consider an infinite slowly convergent 1-D summation $S$ for the series $f$ in (1). Unless an analytic solution for $S$ exists, the limit of the summation operation has to be truncated at a relatively large value, say $N_{t}$, for its numerical evaluation, thus resulting in an approximation as

$$
S=\sum_{n=0}^{\infty} f(n) \simeq S_{N_{t}}=\sum_{n=0}^{N_{t}} f(n) .
$$

For the approximation to be correct, $N_{t}$ is usually chosen very large, particularly for slowly convergent summations. Moreover, if the summation is highly oscillatory, the numerical computation of the summation becomes very laborious and the numerical accuracy becomes questionable for a large value of $N_{t}$. Therefore, even with a very large $N_{t}, S$ may not be obtained accurately via the DS. The proposed method in this study is developed to find an accurate estimate of $S$ without having to incorporate a large number of terms. The idea is based on the use of the GPOF method over a set of partial sums

$$
\mathbf{S}=\left\{S(1), S(2), \ldots, S\left(N_{s}\right)\right\}
$$

of a given summation, where the partial sums are obtained recursively as

$$
\begin{aligned}
S(m)= & \sum_{n=0}^{N_{b}+m N_{a}-1} f(n) \equiv S(m-1) \\
& +\sum_{n=N_{b}+(m-1) N_{a}}^{N_{b}+m N_{a}-1} f(n), \quad m=1, \ldots, N_{s} .
\end{aligned}
$$

$N_{s}$ is the number of partial sums, $N_{a}$ is the number of new terms added to each previous partial sum in $\mathbf{S}$, and $N_{b}$ is the number of bias terms to evaluate $S(0)=\sum_{n=0}^{N_{b}-1} f(n)$. The use of the bias may improve the convergence of the method for some summations, for which the partial sums show their convergence nature beyond certain number of terms, i.e., beyond the transient region. However, for summations/integrals with no knowledge of their transient behaviors, $N_{b}$ can be simply set to zero.

Note that, since the partial sums can be obtained recursively, as shown in (3), $\mathbf{S}$ is computed by adding only $\left(N_{a} \times N_{s}\right)+$ $N_{b}$ terms and can be considered as a function of $m$ for constant $N_{a}$. It is worth to note that the number of terms employed by the proposed method $\left[\left(N_{a} \times N_{s}\right)+N_{b}\right]$ is in general far less than the number of terms needed by the direct sum $\left(N_{t}\right)$ for the same level of convergence. If the partial sums are used as the samples to be input for the GPOF method, $\mathbf{S}$ versus $m$ can be approximated in terms of $M$ complex exponentials as

$$
S(m) \cong \sum_{i=1}^{M} b_{i} e^{s_{i} m}
$$

where $b_{i} \mathrm{~s}$ are the complex residues and $s_{i} \mathrm{~s}$ are the complex poles. Thus, the limiting value of $\mathbf{S}$ as $m \rightarrow \infty$ yields two possibilities:

1) If $\mathbf{S}$ is divergent, then at least one of the complex poles has a positive real part, i.e., $\Re\left\{s_{i}\right\}>0$ for at least one $i$ where $i=1, \ldots, M$.

2) If $\mathbf{S}$ is convergent, then all except one complex poles have negative real parts and the one pole is zero, i.e., $\Re\left\{s_{i}\right\}_{i \neq k}<0$ for $i=1,2, \ldots, k-1, k+1, \ldots, M$ and $s_{k}=0$. Hence, the complex residue corresponding to the zero pole, $b_{k}$, has to be the result of the summation $S$, as the partial sums of a convergent summation would eventually converge to a constant value, i.e., to the sum.

\section{A. Slowly Oscillating Summations}

It is important to note that the efficiency of the algorithm is strongly dependent on the behavior of $\mathbf{S}$ versus $m$ and thus $N_{a}$; however, the relation between the behavior of $f(n)$ and $S(m)$ is not always obvious. For a general rule of thumb, it would be better if the set of partial sums covers at least half a period of $\mathbf{S}$ versus $m$ variation. However, if $\mathbf{S}$ versus $m$ variation is very slowly oscillating, a half period of $\mathbf{S}$ versus $m$ curve may require evaluation of the partial sums for a large number of terms in (3), $N_{a} \times N_{s}$. A possible remedy for this problem may be to accelerate the evaluation of partial sums using the GPOF method as follows: let the partial sums to be evaluated be available up to $S\left(N_{s}\right)$ in (3), then, the partial sums for $N \geq N_{s}$ can be written as

$$
S_{N}=S\left(N_{s}\right)+\sum_{n=N_{b}+N_{s} N_{a}}^{N} f(n) .
$$

Making use of the GPOF method over a set of $f(n)$ for the second term in (5), $f(n)$ can be approximated in terms of $M_{1}$ complex exponentials as

$$
f(n) \simeq \sum_{l=1}^{M_{1}} b_{l} e^{s_{l} \delta\left(n-N_{b}-N_{a} N_{s}\right)} ; \quad n \geq N_{b}+N_{a} \times N_{s}
$$

where $\delta$ is the sampling period, $b_{l}$ s and $s_{l}$ s are the coefficients and the exponents of the approximation, respectively. Then, substituting (6) into (5), changing the order of summations, and evaluating the inner summation over $n$ analytically, $S_{N}$ can simply be expressed as

$$
S_{N}=S\left(N_{s}\right)+\sum_{l=1}^{M_{1}} b_{l} \frac{1-e^{s_{l} \delta\left(N-N_{b}-N_{a} N_{s}+1\right)}}{1-e^{s_{l} \delta}} .
$$

Provided that $S\left(N_{s}\right)$ was calculated before, (7) can be used to obtain the set of partial sums very efficiently. 


\section{B. Algorithm}

Based on these principles and under the assumption that no a priori information is known about the behavior of $\mathbf{S}$ versus $m$ [as well as on $f(n)$ ], an iterative algorithm is proposed as follows.

Step 1) Predefine your error $|e|$, initialize $N_{s}, N_{b i g}\left(>N_{a} \times\right.$ $N_{s}$ that will be used to decide if the $\mathbf{S}$ versus $m$ variation is fast or slowly oscillating).

Step 2) During the calculation of the partial sums, predict the period of the $\mathbf{S}$ versus $m$ variation, and in turn, $N_{a}$ and $N_{s}$ can be determined. Consequently, one can decide if the $\mathbf{S}$ versus $m$ variation is fast or slow, Step 3) based on comparing the predicted period with $N_{\text {big }}$.

a) Fast oscillating:

i) Use (3) to form a set of partial sums.

b) Slowly oscillating:

i) Determine or provide an educated guess, if possible, for the period of $f(n)$.

ii) Use (5)-(7) to form a set of partial sums. Note that because (7) is a closed-form expression for the partial sums, any partial sum required can be evaluated analytically.

Step 4) Use (4) to find $b_{k}^{j}$, where $j$ denotes the number of iteration of the algorithm.

Step 5) Check if

$$
\frac{\left|b_{k}^{j}-b_{k}^{j-1}\right|}{\left|b_{k}^{j}\right|}<|e|
$$

a) If (8) is satisfied, our final sum is $b_{k}^{j}$.

b) If (8) is not satisfied, for the fast oscillating case, go to Step 3(a) to form a new set of partial sums using (3) starting with $n=N_{b}+(j-1) N_{s} N_{a}$.

c) If (8) is not satisfied for the slowly oscillating case, go to Step 3(b)(ii) and use (7) to form a new set of partial sums for even larger values of $\mathrm{N}$.

Note that although the algorithm proposed here works well for all cases considered in this study, it is by no means a unique algorithm.

\section{Extension to 2-D Problems}

The same idea can be directly extended to the 2-D summations because once the partial sums are obtained to be used in the GPOF method, the rest of the procedure would be the same. Let us consider an infinite 2-D summation as

$$
S=\sum_{i=0}^{\infty} \sum_{j=0}^{\infty} f(i, j)
$$

where $f$ is a function of two variables and the partial sums can be computed recursively as

$$
S(m)=S(m-1)+\sum_{i=N_{b 1}+(m-1) N_{a 1}}^{N_{b 1}+m N_{a 1}-1} \sum_{j=0}^{N_{b 2}+m N_{a 2}-1} f(i, j)
$$

$$
+\sum_{i=0}^{N_{b 1}+(m-1) N_{a 1}-1} \sum_{j=N_{b 2}+(m-1) N_{a 2}}^{N_{b 2}+m N_{a 2}-1} f(i, j) m=1, \ldots, N_{s}
$$

starting from $S(0)=\sum_{i=0}^{N_{b 1}-1} \sum_{j=0}^{N_{b 2}-1} f(i, j)$, where $N_{b 1}$ and $N_{b 2}$ are the bias numbers, $N_{a 1}$ and $N_{a 2}$ correspond to the sampling intervals for two dimensions. Once the $S(m)$ values are computed, they can be approximated in terms of complex exponentials, and similarly, the residue of the exponential term with zero complex pole corresponds to the result of the summation.

\section{NUMERICAL RESUlts AND Discussions}

\section{A. 1-D Examples}

1) Summations: The first example for 1-D infinite summations is related with the addition theorem of Hankel functions [15] given by

$$
H_{0}^{(2)}\left(k_{\rho}\left|\bar{\rho}-\bar{\rho}^{\prime}\right|\right)=\sum_{n=0}^{\infty} \kappa_{n} H_{n}^{(2)}\left(k_{\rho} \rho\right) J_{n}\left(k_{\rho} \rho^{\prime}\right) \cos (n \Delta \phi)
$$

which is widely used in cylindrical geometries. This particular form given in (11) is used in the development of closedform Green's function (CFGF) representations of cylindically stratified media [7]. Its right-hand side is computed using the proposed method and is compared with the analytical result $H_{0}^{(2)}\left(k_{\rho}\left|\bar{\rho}-\bar{\rho}^{\prime}\right|\right)$ to assess the accuracy and efficiency of the proposed summation method. In (11), $\rho^{\prime}$ and $\rho$ are the radial distances of the source and observation points, respectively, from the central axis of the cylinder and are selected to be equal to each other (i.e., $\rho=\rho^{\prime}$ ). This case is usually used in mutual coupling problems, and its evaluation poses difficulties due to its fast oscillating and slowly converging behavior. Besides, in (11), $\Delta \phi=\phi-\phi^{\prime},\left|\bar{\rho}-\overline{\rho^{\prime}}\right|=\sqrt{\rho^{2}+\rho^{\prime 2}-2 \rho \rho^{\prime} \cos (\Delta \phi)}$, $k_{\rho}=\sqrt{k^{2}-k_{z}^{2}}$ with $k=2 \pi / \lambda$ and $k_{z}=0$. Note that $k_{z}$ changes between 0 and $k_{z \infty}$ (which is a numerically large $k_{z}$ value along the real $k_{z}$ axis [7]) in the course of finding the CFGF expressions, the difficulty in the summation appears when $k_{\rho}$ is real. Hence $k_{z}=0$, the worst convergence is taken into consideration. Finally, $\kappa_{n}=1$ when $n=0$, and $\kappa_{n}=2$ for all other $n$ values.

Selecting $\rho=\rho^{\prime}=3 \lambda$ with $\lambda=1 \mathrm{~m}$, the analytical value of $H_{0}^{(2)}\left(k_{\rho}\left|\bar{\rho}-\bar{\rho}^{\prime}\right|\right)$ is $-0.16422-\mathrm{j} 0.20284$ when $\Delta \phi=0.5$, and it becomes $0.99777+\mathrm{j} 1.57204$ when $\Delta \phi=0.005$. Denoting

$$
S_{N_{t}}=\sum_{n=0}^{N_{t}} \kappa_{n} H_{n}^{(2)}\left(k_{\rho} \rho\right) J_{n}\left(k_{\rho} \rho^{\prime}\right) \cos (n \Delta \phi)
$$

the imaginary parts of $S_{N_{t}}$ versus $N_{t}$ are plotted for $\Delta \phi=0.5$ and $\Delta \phi=0.005$, in Figs. 1 and 2, respectively.

Note that, the oscillatory nature of the summation (with respect to $N_{t}$ ) given in (12) is due to its imaginary part (the real part does not show such a variation). Also note that larger $\Delta \phi$ improves the convergence but exhibits a faster oscillatory 


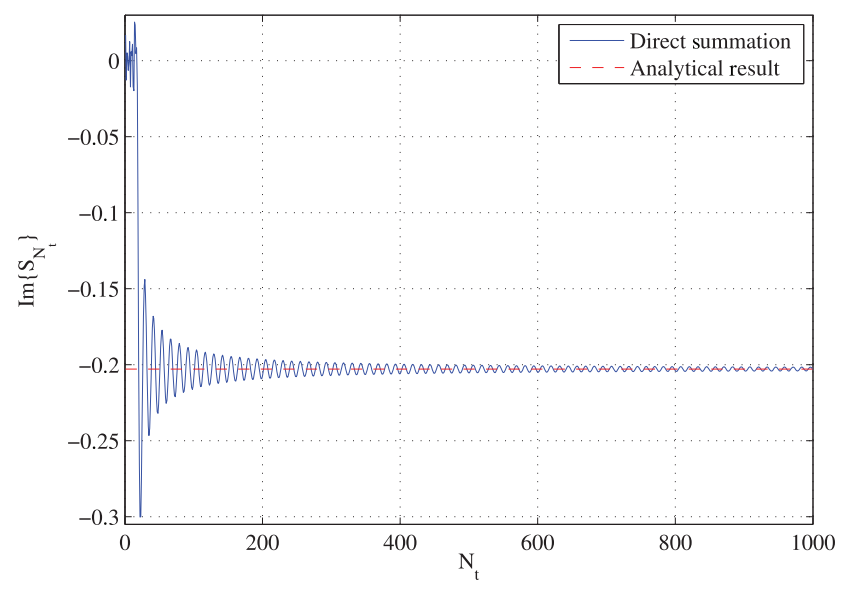

Fig. 1. Imaginary part of $S_{N_{t}}$ versus $N_{t}$ for $\Delta \phi=0.5$.

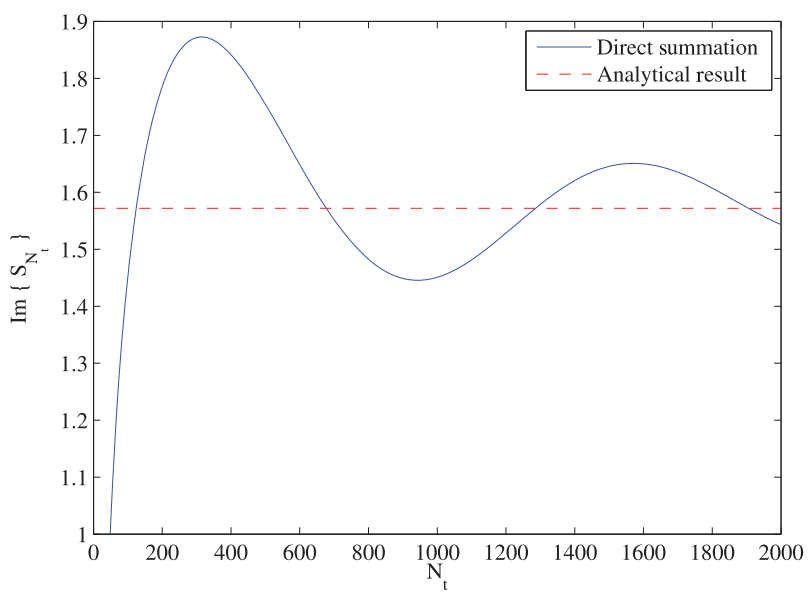

Fig. 2. Imaginary part of $S_{N_{t}}$ versus $N_{t}$ for $\Delta \phi=0.005$.

TABLE I

Number of TERMS FOR THE Algorithms (DS, ST, AND PA) FOR THREE-ERROR LEVELS

\begin{tabular}{|c|c|c|c|}
\hline \multirow{2}{*}{ Error: $|e|$} & \multicolumn{3}{|c|}{ Number of terms $\left(N_{t}\right)$} \\
\cline { 2 - 4 } & $\mathrm{DS}$ & $\mathrm{ST}$ & $\mathrm{PA}$ \\
\hline $10^{-3}$ & $\approx 6000$ & $\approx 4500$ & $75\left(n_{i t}=3\right)$ \\
\hline $10^{-4}$ & $\approx 60000$ & $\approx 45000$ & $90\left(n_{i t}=4\right)$ \\
\hline $10^{-5}$ & $>100000$ & Not applicable & $120\left(n_{i t}=6\right)$ \\
\hline
\end{tabular}

For PA: $N_{b}=30, N_{a}=1, N_{s}=15, N_{t}=N_{b}+n_{i t} N_{s} N_{a}, n_{i t}$ : no. of iterations.

nature. Therefore, fast oscillating part of the algorithm must be used for $\Delta \phi=0.5$. The algorithm is checked for three different error levels, and the numbers of summed terms required to satisfy these error levels for DS, ST, and the proposed algorithm (PA) are summarized in Table I for Fig. 1. The significant reduction in the required number of terms achieved by the PA can be easily observed from Table I. In addition, the computational times of DS, ST, and PA are provided in Table II for three-error levels. As seen in Table II, the proposed approach is computationally more efficient than the DS and the ST.

On the other hand, in Fig. 2, although only $S_{N_{t}}$ up to $N_{t}=2000$ is given, the DS does not converge to the analytical result even for $N_{t}=100000$ when $|e|=10^{-3}$ is selected ( $N_{t} \sim 70000$ for ST). Consequently, $\Delta \phi=0.005$ corresponds
TABLE II

EFFICIENCY OF THE ALGORITHMS (DS, ST, AND PA) FOR THREE-ERROR LEVELS

\begin{tabular}{|c|l|c|c|}
\hline \multirow{2}{*}{ Error: $|e|$} & \multicolumn{3}{|c|}{ CPU times } \\
\cline { 2 - 4 } & DS & ST & PA \\
\hline $10^{-3}$ & 0.228431 & 0.129657 & 0.075160 \\
\hline $10^{-4}$ & 2.159839 & 1.118095 & 0.075819 \\
\hline $10^{-5}$ & $>10$ & Not applicable & 0.077413 \\
\hline
\end{tabular}

to a slowly oscillating case. Hence, slowly oscillating part of the algorithm is applied for this case. After a transient part, which contains approximately 300 terms, the algorithm uses (5)-(7) to find the partial sums efficiently as follows: first, the argument of the summation $f(n)=\kappa_{n} H_{n}^{(2)}\left(k_{\rho} \rho\right) J_{n}\left(k_{\rho} \rho^{\prime}\right) \cos (n \Delta \phi)$ is approximated in terms of $M_{1}=8$ complex exponentials from roughly 50 samples of $f(n)$. Then, approximately 50 samples of $S_{N}$ corresponding to very large value of $N\left(N \gg N_{b i g}\right)$ are obtained using (7) and are approximated in terms of 7 complex exponentials in each iteration. In 3 and 4 iterations, the final result is achieved when $|e|=10^{-3}$ and $|e|=10^{-5}$, respectively.

The second 1-D example is related to the surface fields of an impedance sphere with a radius $a$. The $H_{\theta}(\bar{r})$ component of a surface magnetic field, excited by a tangential magnetic source, $\bar{M}=\hat{x} p_{m} \delta\left(\bar{r}-\bar{r}^{\prime}\right)$, located on the sphere $\left(r^{\prime}=a, \theta^{\prime}=\right.$ $\left.0, \phi^{\prime}=0\right)$, is given by [8]

$$
H_{\phi}=\sin \phi \frac{k^{2} Y_{0} p_{m}}{4 \pi} \sum_{n=1}^{\infty}\left[S_{1}(n)+S_{2}(n)\right]
$$

when the field point is on the surface of the sphere (i.e., $r=$ $a, \theta, \phi)$. In (13), $S_{1}(n)$ and $S_{2}(n)$ are the problematic summations, which are not absolutely convergent, and are given by

$$
\begin{aligned}
S_{1}(n)= & \frac{2 n+1}{n(n+1)} \frac{j}{(k a)^{2}}\left[-j \Lambda+\frac{(n+1)}{k a}\right. \\
& \left.-\frac{h_{n+1}^{(2)}(k a)}{h_{n}^{(2)}(k a)}\right]^{-1} \frac{\partial P_{n}^{1}(\cos \theta)}{\partial \theta} \\
S_{2}(n)= & \frac{2 n+1}{n(n+1)} \frac{j}{(k a)^{2}}\left(\frac{-1}{\sin \theta}\right) \\
& {\left[\frac{\frac{(n+1)}{k a}-\frac{h_{n+1}^{(2)}(k a)}{h_{n}^{(2)}(k a)}}{1+j \Lambda\left[\frac{(n+1)}{k a}-\frac{h_{n+1}^{(2)}(k a)}{h_{n}^{(2)}(k a)}\right]}\right] P_{n}^{1}(\cos \theta) }
\end{aligned}
$$

where $h_{n}^{(2)}$ is the spherical Hankel function, $P_{n}^{1}(\cos \theta)=$ $\frac{\partial}{\partial \theta} P_{n}(\cos \theta)$ with $P_{n}$ being the usual Legendre function, $k=2 \pi / \lambda, \Lambda=Z_{s} / Z_{0}$ is the normalized surface impedance with $Z_{s}, Z_{0}$, and $Y_{0}$ being the surface impedance, free-space impedance, and free-space admittance, respectively. Finally, $p_{m}$ represents the strength of the magnetic current.

The magnitudes and phases of $H_{\phi}$ component of tangential magnetic field calculated with the proposed summation method and with the DS for various $N_{t}$ values are plotted in Fig. 3 for $a=3 \lambda, \Lambda=0.75, \phi=\pi / 2$, and $\theta$ varying from $\pi / 6$ to $5 \pi / 12$. 

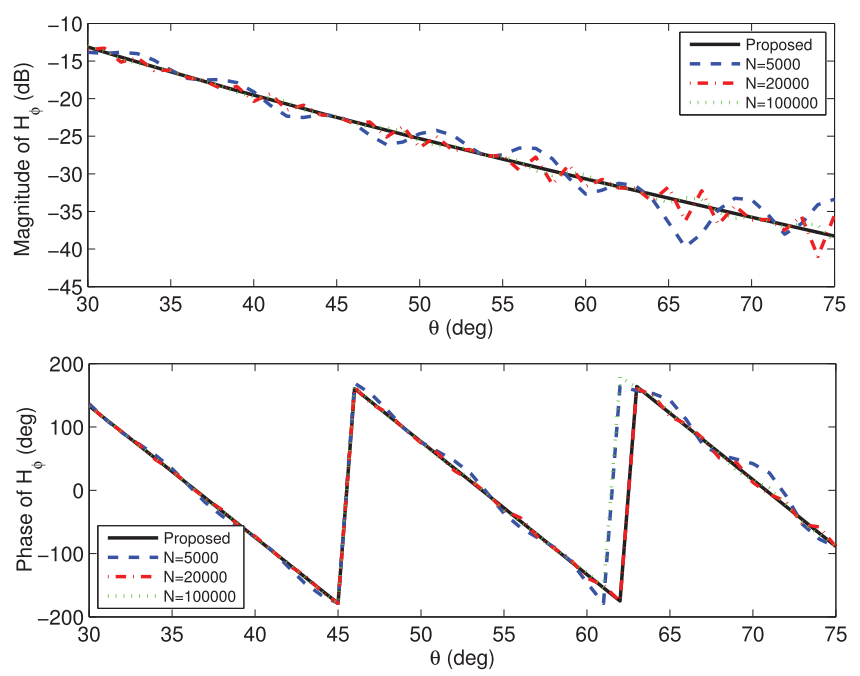

Fig. 3. Comparison of magnitudes and phases of $H_{\phi}$ component of tangential magnetic field calculated with the proposed summation method and with the DS for various $N_{t}$ values when $a=3 \lambda, \Lambda=0.75, \phi=\pi / 2$, and $\theta$ varying from $\pi / 6$ to $5 \pi / 12$.

Very accurate and converged result is obtained using the proposed summation method by using 20 samples and 7 complex exponentials after approximately 50 terms of transient part that requires overall 3 iterations for $|e|=10^{-3}$. However, the results (both magnitude and phase of $H_{\phi}$ ) do not converge even for $N_{t}=100000$ and become worse if more terms are added as the summation is not absolutely convergent.

2) Integrals: The spatial-domain Green's functions $G$ for stratified media are evaluated from their spectral-domain counterparts $\tilde{G}$ via the Sommerfeld integral $(S I)$

$$
G(\rho)=\frac{1}{2 \pi} \int_{0}^{\infty} \tilde{G}\left(k_{\rho} ; z, z^{\prime}\right) J_{0}\left(k_{\rho} \rho\right) k_{\rho} d k_{\rho}
$$

where $J_{0}$ is a zeroth-order Bessel function of the first kind, and $\rho$ is the lateral distance between the observation and the source points with their corresponding vertical coordinates $z$ and $z^{\prime}$, respectively. The problems of efficient evaluation of $S I$ s arise from the possible singularities and the oscillatory and slowly decaying nature of the integrands. These two problems can be solved independently by dividing the integration path into two parts

$$
G=\frac{1}{2 \pi}\left(I_{1}\left(0, \xi_{0}\right)+I_{2}\left(\xi_{0}, \infty\right)\right)
$$

where $\xi_{0}$ should be properly selected to ensure that all singularities lie in $I_{1}$, and consequently $I_{2}$, also known as the $S I$ tail, becomes free of singularities. Although the evaluation of $I_{1}$ is out of the scope of this study, for the sake of completeness, it should be noted that $I_{1}$ can be computed by subtracting the singularities from the integrand by using Cauchy integration formula and then numerically integrating the remaining part. At the final step, the contribution of the singularities is added in the spatial domain. In this paper, the $S I$ tail examples are evaluated by the proposed method, ST, and the WA extrapolation technique, and all numerical integrations are computed using adaptive Gauss-Kronrod quadrature in MATLAB programming language. In order to apply these methods, the $S I$

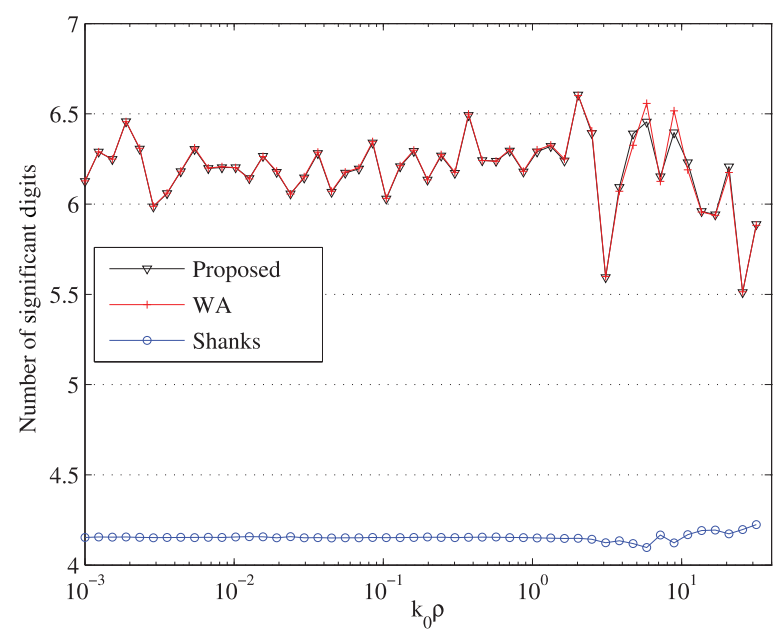

Fig. 4. Comparison of the three methods on the computation of the SI (19). Number of significant digits obtained by using 12 partial integrals.

tail is expressed as an infinite sum of partial integrals over the finite subintervals

$$
I_{2}=\sum_{i=0}^{\infty} \int_{\xi_{i}}^{\xi_{i+1}} \tilde{G}\left(k_{\rho} ; z, z^{\prime}\right) J_{0}\left(k_{\rho} \rho\right) k_{\rho} d k_{\rho} .
$$

In this paper, the equidistant integral boundaries are selected as $\pi / \rho$, i.e., the asymptotic half periods of Bessel function, since much simpler and more efficient expressions can be obtained for the generalized WA algorithm if the equidistant break points are separated by half periods [12]. In addition, the built-in functions in MATLAB are used for the evaluation of the binomial coefficients to obtain the weights of the WA algorithm.

In order to start with a controllable numerical experiment, the tail of the Sommerfeld identity, with its known analytical expression (19), at $z=0$, which is the most slowly converging case, has been examined first

$$
\int_{0}^{\infty} \frac{e^{-j k_{z}|z|}}{j k_{z}} J_{0}\left(k_{\rho} \rho\right) k_{\rho} d k_{\rho}=\frac{e^{-j k r}}{r}
$$

where $k_{z}=\sqrt{k^{2}-k_{\rho}^{2}}$. The accuracy of the proposed method, and the other methods that are used for the purpose of comparison, is assessed by the number of significant digits, as computed by $-\log _{10}$ |relative error|, using 12 partial integrals. It is observed that the WA algorithm and the proposed method provide almost identical results and significantly better than the results obtained by the ST, as depicted in Fig. 4.

To further understand the dynamics of the methods compared, Fig. 5 provides information on the increase in the number of significant digits as the number of partial sums for a given value of $\rho$. It is observed that the WA algorithm achieves the highest accuracy even with a few number of partial integrals, while the proposed method achieves the same accuracy after the nine partial integrals. Meanwhile, the ST requires a very high number of partial integrals to achieve such an accuracy. However, for the efficiency of the methods, the computation times required for the ST and the proposed approach are not 


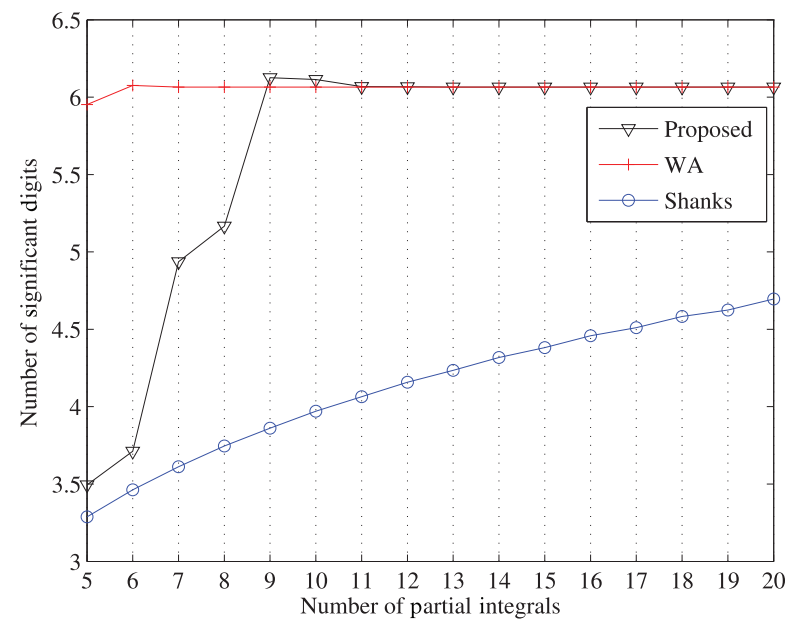

Fig. 5. Sommerfeld identity (19): the number of significant digits versus number of partial integrals for $k_{0} \rho=0.1$.

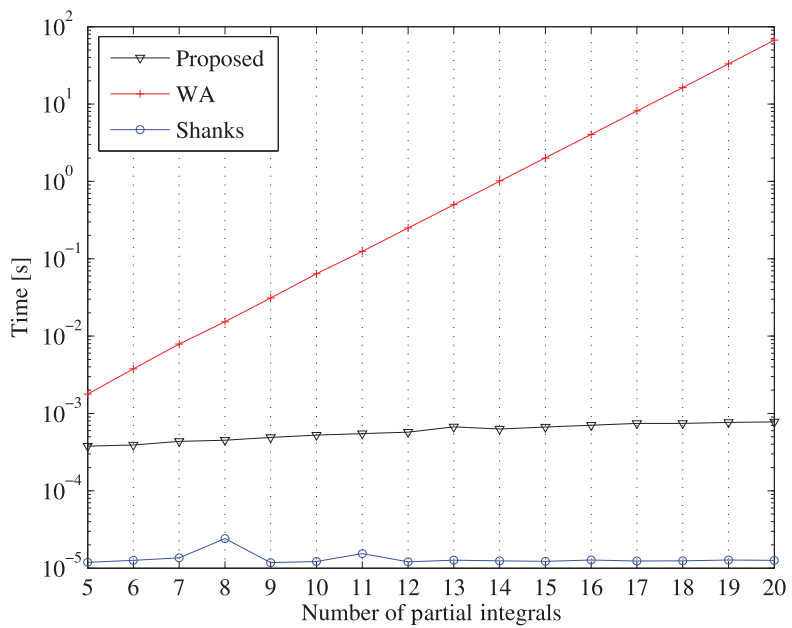

Fig. 6. Sommerfeld identity (19): computation time versus number of partial integrals for $k_{0} \rho=0.1$.

affected critically by the increase in the number of partial sums, while the computation time of the WA algorithm seems quite sensitive due to the computation of the binomial coefficients to obtain the corresponding weights as seen in Fig. 6. It is also verified that the behavior of the methods is similar for other $\rho$ values.

To demonstrate the algorithm on a Green's function for a realistic geometry, a lossy slab in free space was considered with a source of an horizontal electric dipole (HED) operating at $f=4 \mathrm{GHz}$ and located at the interface between the upper boundary of the lossy medium and the air, as shown in Fig. 7. The vector and scalar Green's functions for this geometry were evaluated by the same three methods as the ones used for the Sommerfeld identity, providing the results with no distinguishable differences, as shown in Fig. 8. Once the accuracy of the methods has been verified, their comparative efficiencies are assessed by the CPU times of the methods, as provided in Fig. 9. In all techniques, the major time consumption occurs in the evaluation of the partial integrals. Since all three algorithms take the same partial integrals as their inputs, we do not include the partial integral calculations into the time comparison of the

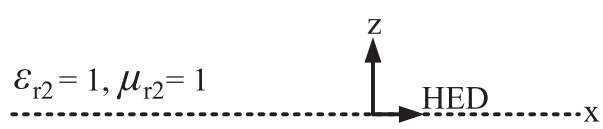

$$
\begin{aligned}
& \varepsilon_{\mathrm{r} 1}=4-0.3 \mathrm{j}, \mu_{\mathrm{r} 1}=1 \quad \mathrm{~d}_{1}=10 \mathrm{~mm} . \\
& \varepsilon_{\mathrm{r} 0}=1, \mu_{\mathrm{r} 0}=1
\end{aligned}
$$

Fig. 7. Lossy dielectric material in air at $f=4 \mathrm{GHz}$.

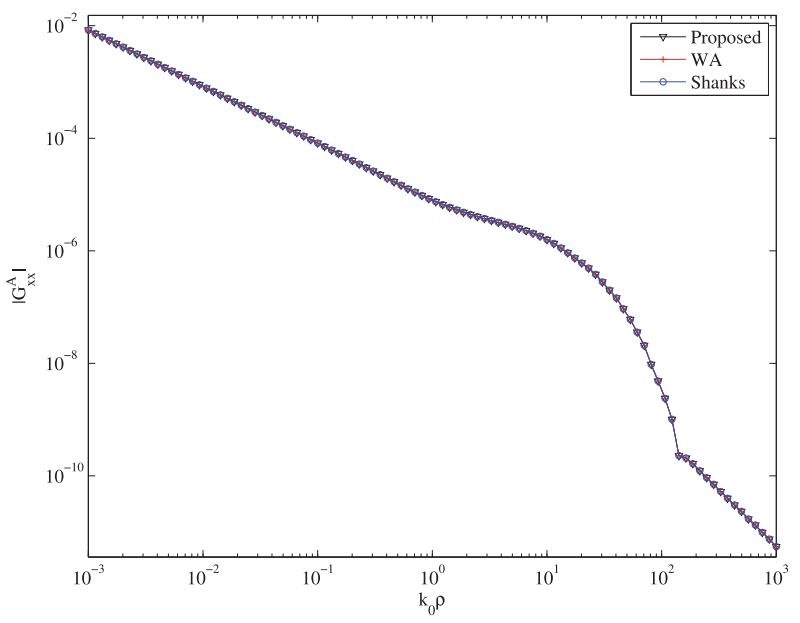

(a)

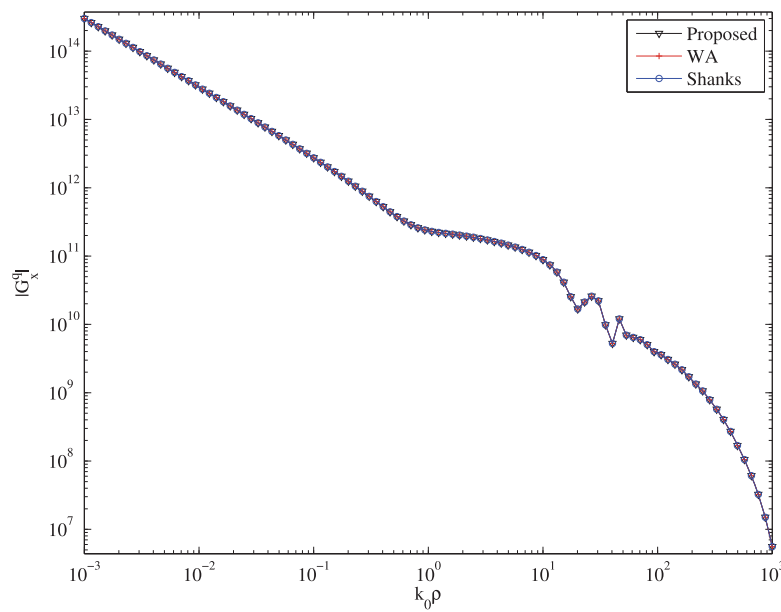

(b)

Fig. 8. Magnitudes of the Green's functions of (a) the vectoral potential and (b) the scalar potential for the geometry in Fig. 7 at $f=4 \mathrm{GHz}$.

algorithms. As seen in Fig. 9, the proposed method is noticeably more efficient than the WA algorithm for the same $S I$ tail.

\section{B. 2-D Example}

To demonstrate the applicability of the proposed method to the efficient evaluation of 2-D series, the slowly convergent free-space periodic Green's functions (FSPGF) for 3-D problems with 2-D periodicity are examined. The efficient and accurate computation of the periodic Green's function plays an important role in the method-of-moments (MoM) analysis of periodic structures. The construction of the MoM matrix 


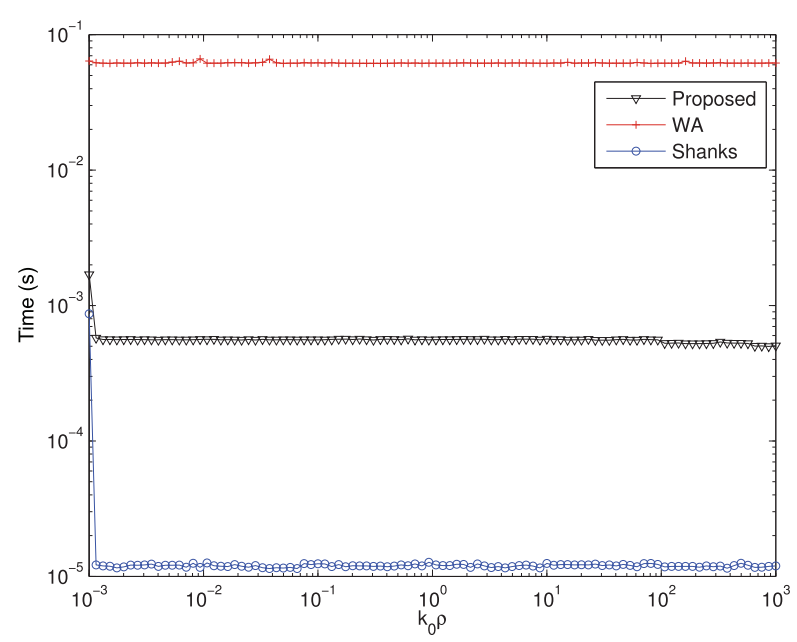

Fig. 9. Time comparison for the spatial domain vector Green's function of a lossy dielectric slab in air at $f=4 \mathrm{GHz}$.

requires repeated evaluations of the FSPGF for various values of the source and the observation points. Therefore, the matrix fill time increases dramatically unless the slowly convergent series appearing in the FSPGF expression are computed efficiently through the use of an acceleration technique. A number of techniques have been developed in the past to accelerate the convergence of the series, and a comparative analysis of these acceleration techniques can be found in [16]. As discussed in [16], the most commonly used acceleration techniques are Kummer decomposition combined with ST [11] and Ewald's transformation [14]. Ewald's transformation can be considered as the most efficient method especially for 3-D problems with 2-D periodicity. Hence, the proposed method will be compared to the Ewald's and ST regarding the accuracy and the computation time.

Since the proposed method is utilized in the evaluation of the FSPGF after performing Kummer decomposition, first a brief outline of this decomposition method will be presented. Consider a 2-D periodic array of point sources in the $x-y$ plane with periods $a$ and $b$ and phase shifts $k_{x 0}$ and $k_{y 0}$ along the $x$ - and $y$-axes, respectively. The FSPGF corresponding to this geometry can be written in terms of the following spatial series:

$$
G(\mathbf{r})=\sum_{m=-\infty}^{\infty} \sum_{n=-\infty}^{\infty} e^{j \mathbf{k}_{t 00} \cdot \mathbf{r}_{m n}}\left(\frac{e^{-j k R_{m n}}}{4 \pi R_{m n}}\right)
$$

where $k=2 \pi / \lambda, R_{m n}$ is the distance between the observation point at $\mathbf{r}=(x, y, z)$ and the $(m, n)$ th periodic source point at $\mathbf{r}_{m n}=(m a, n b, z), k_{t 00}=\left(k_{x 0}, k_{y 0}, 0\right)$ is the transverse phasing wave vector. By using Poisson's formula, this spatial series can be transformed to the following spectral series which has a relatively rapid convergence

$$
G(\mathbf{r})=\frac{1}{A} \sum_{p=-\infty}^{\infty} \sum_{q=-\infty}^{\infty} \frac{1}{2 j k_{z p q}} e^{-j k_{z p q}\left|z-z^{\prime}\right|} e^{-j \mathbf{k}_{t p q} \cdot \mathbf{r}}
$$

where $A$ is the cross-sectional area of each lattice cell, $\mathbf{k}_{t p q}=$ $\left(k_{x 0}+\frac{2 \pi p}{a}, k_{y 0}+\frac{2 \pi q}{b}, 0\right)$ is the transverse wavenumber, and $k_{z p q}=\sqrt{k^{2}-\left|\mathbf{k}_{t p q}\right|^{2}}$. Since the series in (21) converges faster than the series in (20), DS and further acceleration techniques are usually applied to (21). The Kummer decomposition relies on the fact that the summation in (21) can be accelerated by subtracting the asymptotic behavior of the series as $p$ and $q$ tend to infinity. By defining a parameter $u$ such that $k_{p q}^{2}=\left|\mathbf{k}_{t p q}\right|^{2}+$ $u^{2}$, the following asymptotic expression can be obtained:

$$
\lim _{p, q \rightarrow \infty}\left(\frac{1}{j k_{z p q}} e^{-j k_{z p q}\left|z-z^{\prime}\right|}\right)=\frac{1}{k_{p q}} e^{-k_{p q}\left|z-z^{\prime}\right|} .
$$

By subtracting (22) from each term of the summation in (21) and then by adding the series corresponding to the asymptotic expression, the series in (21) can be decomposed into two series. Furthermore, the series corresponding to the asymptotic expression can be transformed into a highly convergent series in the spatial domain by applying Poisson's formula. After applying Kummer decomposition and Poisson's formula, the series in (21) is decomposed into the following form [17]:

$$
\begin{aligned}
G(\mathbf{r})= & \frac{1}{2 A} \sum_{p=-\infty}^{\infty} \sum_{q=-\infty}^{\infty}\left[\frac{e^{-j k_{z p q}\left|z-z^{\prime}\right|}}{j k_{z p q}}-\frac{e^{-k_{p q}\left|z-z^{\prime}\right|}}{k_{p q}}\right] e^{-j \mathbf{k}_{t p q} \cdot \mathbf{r}} \\
& +\frac{1}{4 \pi} \sum_{m=-\infty}^{\infty} \sum_{n=-\infty}^{\infty} e^{j \mathbf{k}_{t 00} \cdot \mathbf{r}_{m n}} \frac{e^{-u R_{m n}}}{R_{m n}}
\end{aligned}
$$

The second series in (23) is rapidly converging due to the attenuating factor $u$, and a few number of terms are enough to evaluate this series accurately. Although the convergence of the first series in (23) is accelerated by subtracting the asymptotic term, it still needs to be accelerated. Generally, ST is used to further accelerate this series. Here, instead of ST, the proposed method is used to efficiently compute the first series, and their performances are compared with the Ewald method.

When $\left|z-z^{\prime}\right|=0$, the summation converges only algebraically (not exponentially), and therefore, this worst case is taken as the example in this study. We consider an example at $f=1 \mathrm{GHz}$ with a square lattice of dimension $a=b=0.6 \lambda$ and $\left|z-z^{\prime}\right|=0$. The source is assumed to be at the center of the unit cell $(x=y=0)$, and the FSPGF is evaluated at different observation points along the diagonal $(x=y)$ of the unit cell. First, Kummer decomposition is applied, then the ST and the proposed method are used for the computation of the first series in (23). In Fig. 10, the computation times of two methods and the Ewald method that are obtained for a relative error of $10^{-4}$, are compared. Since the analytical solution is not available for the example, a reference result is obtained by applying the Ewald method until machine precision is reached. The results are then compared with the reference one. It can be observed from Fig. 10 that the proposed method is more efficient than the ST and Ewald method outperforms the two others. However, it should be noted that the proposed method is used in the computation of the FSPGF in order to demonstrate its applicability to the evaluation of 2-D series, not to compete with the computational efficiency of Ewald method. Moreover, Ewald method can be used to accelerate the convergence of a limited set of series satisfying certain properties, whereas the proposed method is versatile. 


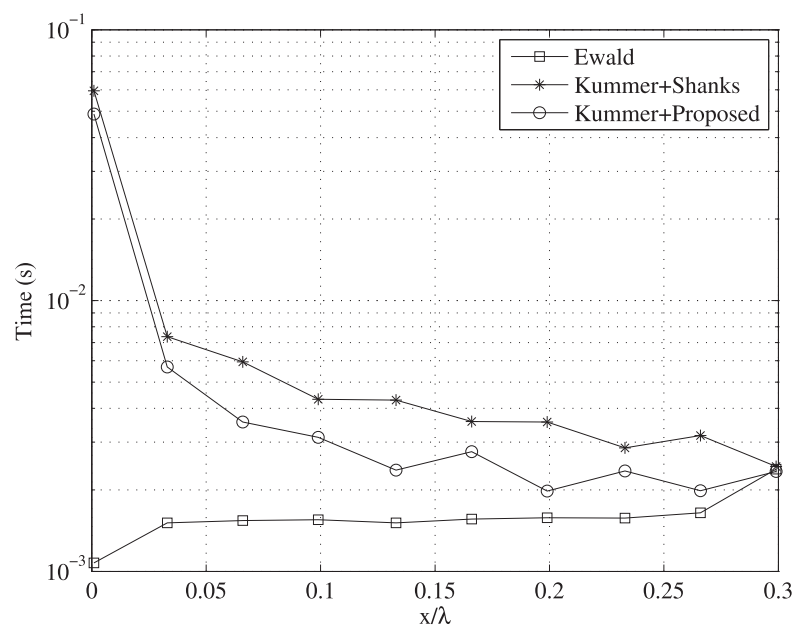

Fig. 10. Computation time comparison of three methods wrt. relative error of $10^{-4}$ for the calculation of 3-D FSPGF with 2-D periodicity evaluated on the diagonal of the unit cell, $\left|z-z^{\prime}\right|=0$ and $a=b=0.6 \lambda$.

\section{CONCLUSION}

In this study, we have presented a novel method to evaluate 1-D and 2-D infinite, slowly converging summations and integrals accurately and efficiently. The method is based on the idea that the partial sums of a convergent summation have an oscillating nature culminating in a zero frequency value, which becomes the result of the summation. In order to acquire this value, a series of partial sums is obtained and approximated in terms of complex exponentials, from which the zero frequency term corresponds to the end result. The method is applied to some typical 1-D and 2-D examples taken from different EM problems for the efficiency and accuracy verification.

\section{REFERENCES}

[1] M. C. Payne, M. P. Teter, D. C. Allan, T. A. Arias, and J. D. Joannopoulos, "Iterative minimization techniques for ab initio total-energy calculations: Molecular dynamics and conjugate gradients," Rev. Mod. Phys., vol. 64, no. 4, pp. 1045-1097, Oct. 1992.

[2] D. Wolf, P. Keblinski, S. R. Phillpot, and J. Eggebrecht, "Exact method for the simulation of Coulombic systems by spherically trancated, pairwise $r^{-1}$ summation," J. Chem. Phys., vol. 110, no. 17, pp. 8254-8282, May 1999.

[3] N. Kinayman and M. I. Aksun, "Comparative study of acceleration techniques for integrals and series in electromagnetic problems," Radio Sci., vol. 30, no. 6, pp. 1713-1722, Nov. 1995.

[4] M.-J. Park and S. Nam, "Rapid calculation of the Green's function in the shielded planar structures," IEEE Microw. Guided Wave Lett., vol. 7 , no. 10 , pp. 326-328, Oct. 1997.

[5] J. R. Mosig and A. Alvarez Melcon, "The summation-by-parts algorithm-A new efficient technique for the rapid calculation of certain series arising in shielded planar structures," IEEE Trans. Microw. Theory Techn., vol. 50, no. 1, pp. 215-218, Jan. 2002.

[6] E. J. Weniger, "Nonlinear sequence transformations for the acceleration of convergence and the summation of divergent series," Comput. Phys. Rep., vol. 10, nos. 5-6, pp. 189-373, Dec. 1989.

[7] S. Karan, V. B. Erturk, and A. Altintas, "Closed-form Green's function representations in cylindrically stratified media for method of moments applications," IEEE Trans. Antennas Propag., vol. 57, no. 5, pp. 11581168, Apr. 2009.

[8] B. Alisan and V. B. Erturk, "A high frequency based asymptotic solution for surface fields on a source excited sphere with an impedance boundary condition," Radio Sci., vol. 45, pp. 1-14, 2010.
[9] Z. Sipus, M. Bosiljevac, and Z. Milin Sipus, "Acceleration of series summation encountered in the analysis of conformal antennas," IEEE Antennas Wireless Propag. Lett., vol. 11, pp. 1521-1524, Dec. 2012.

[10] Y. Hua and T. K. Sarkar, "Generalized pencil-of-function method for extracting poles of an EM system from its transient response," IEEE Trans. Antennas Propag., vol. 37, no. 2, pp. 229-234, Feb. 1989.

[11] D. Shanks, "Non-linear transformations of divergent and slowly convergent sequences," J. Math. Phys., vol. 34, pp. 1-42, 1955.

[12] J. Mosig, "The weighted averages algorithm revisited," IEEE Trans. Antennas Propag., vol. 60, no. 4, pp. 2011-2018, Apr. 2012.

[13] R. Golubovic, A. G. Polimeridis, and J. R. Mosig, "Efficient algorithms for computing Sommerfeld integral tails," IEEE Trans. Antennas Propag., vol. 60, no. 5, pp. 2409-2417, May 2012.

[14] P. P. Ewald, "Die Berechnung optischer und elektrostatischer Gitterpotentiale," Annalen der Physik, vol. 369, no. 3, pp. 253-287, 1921.

[15] C. A. Balanis, Advanced Enginering Electromagnetics, 2nd ed. New York, NY, USA: Wiley-Interscience, 1989.

[16] G. Valerio, P. Baccarelli, P. Burghignoli, and A. Galli, "Comparative analysis of acceleration techniques for 2-D and 3-D Green's functions in periodic structures along one and two directions," IEEE Trans. Antennas Propag., vol. 55, no. 6, pp. 1630-1643, Jun. 2007.

[17] S. Singh, W. F. Richards, J. R. Zinecker, and D. R. Wilton, “Accelerating the convergence of series representing the free space periodic Green's function," IEEE Trans. Antennas Propag., vol. 38, no. 12, pp. 1958-1962, Dec. 1990.

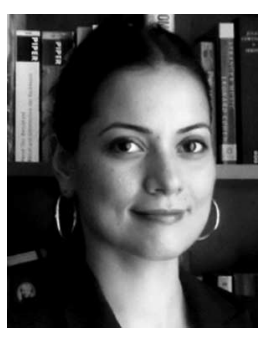

E. Pınar Karabulut (S'06-M'13) received the B.S. degree in electrical and electronics engineering from the Middle East Technical University, Ankara, Turkey, in 2004, the M.S. degree in electrical and computer engineering and the Ph.D. degree in electri$\mathrm{cal}$ and electronics engineering from Koç University, Istanbul, Turkey, in 2006 and 2012, respectively.

Since 2012, she has been with the Department of Electrical and Electronics Engineering, Bahçeşehir University, Istanbul, Turkey. Her research interests include numerical methods for electromagnetics, computational aspects of periodic structures including photonic-bandgap materials and metamaterials, and nanophotonics.

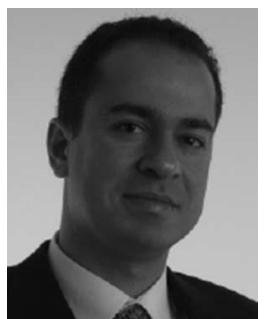

Vakur B. Ertürk (M'00) received the B.S. degree in electrical engineering from the Middle East Technical University, Ankara, Turkey, in 1993, and the M.S. and $\mathrm{Ph} . \mathrm{D}$. degrees from the Ohio-State University (OSU), Columbus, OH, USA, in 1996 and 2000, respectively.

He has been with the Department of Electrical and Electronics Engineering, Bilkent University, Ankara, Turkey. His research interests include the analysis and design of planar and conformal arrays, highfrequency techniques, structural health monitoring, magnetic resonance imaging, scattering from and propagation over large terrain profiles.

Dr. Ertürk was the Secretary/Treasurer of IEEE Turkey Section as well as the Turkey Chapter of the IEEE TRANSACTIONS ON ANTENNAS AND Propagation, iEEE Transactions on Microwave Theory and TECHNIQUES, Electron Devices and Electromagnetic Compatibility Societies. He was the recipient of 2005 URSI Young Scientist and 2007 Turkish Academy of Sciences Distinguished Young Scientist Awards.

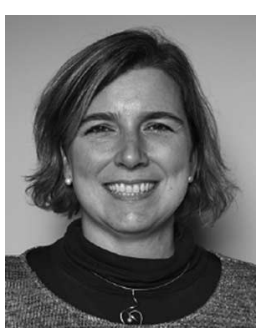

Lale Alatan (S'90-M'97) received the B.Sc., M.Sc., and Ph.D. degrees from the Middle East Technical University (METU), Ankara, Turkey, in 1990, 1993, and 1997, respectively, all in electrical engineering.

Since 2000, she has been with the Department of Electrical and Electronics Engineering, METU. Her research interests include computational methods, optimization techniques, analysis and design of printed antennas, and antenna arrays. 


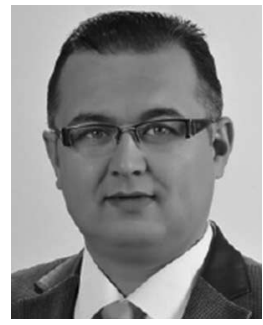

S. Karan received the B.S., M.S., and Ph.D. degrees in electrical and electronics engineering from Bilkent University, Ankara, Turkey, in 2003, 2006, and 2012, respectively.

Since 2003, he has been an RF Design Engineer with the Aselsan Electronics Incorporated, Ankara, Turkey. His research interests include application of numerical methods to radiation and mutual coupling problems associated with cylindrical structures.

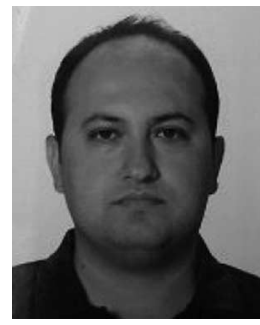

Burak Alisan received the B.S., M.S., and Ph.D. degrees in electrical and electronics engineering from Bilkent University, Ankara, Turkey, in 2003, 2006, and 2012, respectively.

Since 2003, he has been a Radio-Frequency and Microwave Design Engineer with Aselsan Electronics Inc., Ankara, Turkey. His research interests include application of numerical methods and asymptotic high-frequency techniques to radiation and mutual coupling problems associated with convex structures.

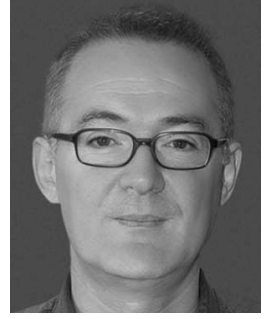

M. I. Aksun (M'92-SM'99) received the B.S. and M.S. degrees in electrical and electronics engineering from the Middle East Technical University, Ankara, Turkey, in 1981 and 1983, respectively, and the $\mathrm{Ph} . \mathrm{D}$. degree in electrical and computer engineering from the University of Illinois at Urbana-Champaign, Champaign, IL, USA, in 1990.

$\mathrm{He}$ is a Professor with the Electrical and Electronics Engineering, Koç University, Istanbul, Turkey. His research interests include numerical methods for electromagnetics and optics, printed circuits and antennas, and photonics.

Dr. Aksun was a Post-Doctoral Fellow with the Electromagnetic Communication Laboratory, University of Illinois at Urbana-Champaign, from 1990 to 1992. From 1992 to 2001, he was on the Faculty with the Department of Electrical and Electronics Engineering, Bilkent University, Ankara, Turkey. In 2001, he was as a Professor with the Faculty with the Department of Electrical and Electronics Engineering, Koç University, and served as the Dean of the College of Engineering during 2004-2009. Starting in September 2009, he has been the Vice President with the Research and Development, Koç University. He received TÜBITAK Encouragement Award in 1994, and TÜBITAK Science Award in 2007. 